\title{
CGA SYNTHESIZER INTERPOLATING AND EXTRAPOLATING MOTION DATA
}

\author{
Kiyoshi Hoshino \\ University of the Ryukyus, and Japan Science \& Technology Corporation
}

\begin{abstract}
The author proposes a computer-graphics animation tool which composes a mixed pattern of two dance motions at any rate, or which interpolates and extrapolates a new dance pattern. Quantitative analyses on human KANSEI, especially on the relationship between human motions and subjective impressions brought about can be carried out through the synthesizer.

A magnetic motion capture system with sixteen sensors and a pair of data gloves with eighteen sensors each were used to obtain various types of dance motions. Time-series changes of each joint angles were estimated first from the motion data, and Fourier transformation was adopted to obtain the spectrum amplitude with DC component and the phase spectrum of each joint. Then, the differences between two kinds of dance motions at each same joints were extracted in the frequency domain, and the differences were increasingly or decreasingly weighted according to users' input score. Finally, every angle motions were synthesized through inverse Fourier transformation with careful operation of the phase information of each joint angles, which is producing and keeping harmony among joint motions in a computer-graphics animation.
\end{abstract}

Key words: computer-graphics animation, interpolation and extrapolation of motions, motions and impressions

\section{Introduction}

For the future man-machine interface system, the ability of transmitting intended feelings, emotions or KANSEI is needed to be realized through the machine movement, as well as the ability of understanding the human feelings expressed through the motions. The author's group, therefore, has

The original version of this chapter was revised: The copyright line was incorrect. This has been corrected. The Erratum to this chapter is available at DOI: 10.1007/978-0-387-35660-0_65 
designed humanoid robotic arms and hands shown in Figures 1 and 2, carrying out the experiments of transmitting subjective impressions and feelings to observers through various kinds of robot motions. Meanwhile, a large number of previous studies has been made on discussion about subjective impressions evoked by movement such as dances, little is known about the relationship between motions and subjective impressions [1]. Our group has been carrying out quantitative analyses of the relationship between motion characteristics and subjective impressions using Okinawan "Kachaasii" dance where people can improvise on their motions and move their arms, hands and legs freely [2]. But the knowledge of the relationship should be clear so much as to almost freely synthesize motions with impressions intended. For example, kinematic or dynamic features which produces subjective impressions such as "dynamic" or "elegant" may be quantitatively analyzed by a so-called motion synthesizer interpolating or extrapolating two kinds of motions at any rate.

There are some defects in a previous system [3] interpolating and extrapolating motions: the rotary motions such as a forearm winding or leg winding is technically immeasurable, and therefore, the system was adaptable only to synthesizing human "walking" which is almost figured out by angles' movement observed just from one side, due to an optical motion capture technique with two video camera and with reflective markers attached to a human subject. In addition, the experimental procedures require a lot of operators' exercise and skill for correction of estimated angles' motions due to indistinct vide images. Moreover, the size of hand and fingers is small, but the structure or the shape greatly changes, and the selfocculusion happens frequently, which disturb the estimation of the minute motions; The accurate measurement, analysis and synthesize of movement of human arms, hands and fingers can hardly be carried out with optical motion capture system.

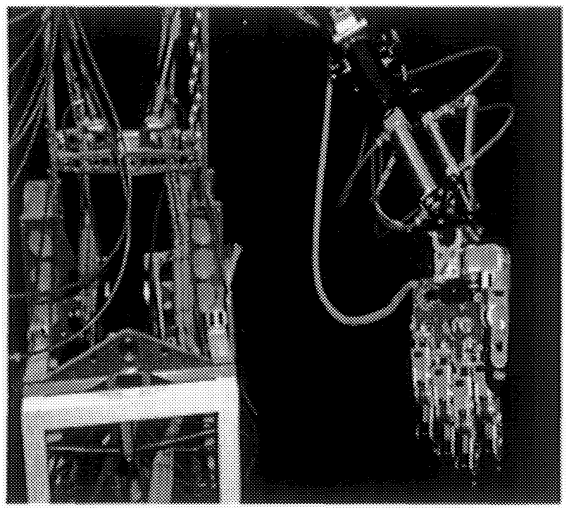

Figure 1.Humanoid robotic arm

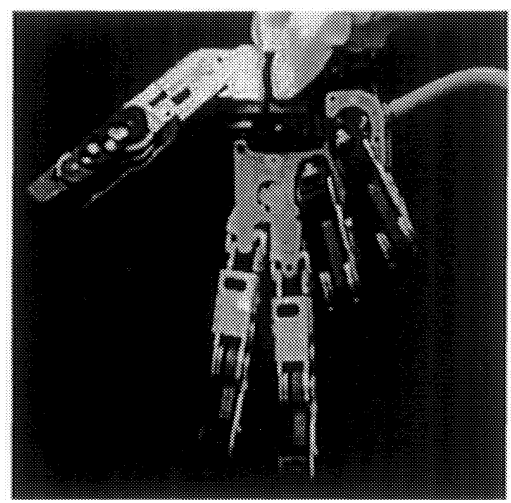

Figure 2.Humanoid robotic hand 
The use of the magnetic motion capture system with data gloves may be one solution for estimating and synthesizing three-dimensional hand and finger motions as well as arm and leg motions, through which the rotary motions can be measured, which is robust for self-occulusion of the body, and which may output the sufficient high frequency data for control of robots. But, the considerable amount of noise is usually overlapped in the system output, suggesting that the appropriate time-series data of the joint angles may not be obtained. In order to reduce the amount of errors due to the system noise, statistically averaged center points of each joint in a certain period of time should be used as well as neighboring two sensors, in stead of using the joint angles obtained with each two sensors neighboring. The use of the joint-centers information can reduce the noise and errors due to the shifts of sensors positions caused by swelling of the human muscles.

In this study, therefore, we propose a new computer-graphic animation tool which can interpolate and extrapolate between two patterns of dance motions, using a wireless magnetic motion capture system with a pair of data gloves and a new estimation algorithm where the time-series joint angle data are estimated with a statistically averaged center points of each joint and neighboring two sensors. The purpose of designing this system is to quantitatively investigate the relationship between human motions and subjective impressions under the condition of little system noise. Through our motion synthesizer, kinematic or dynamic features which may produce various impressions to the human movement are possibly analyzed by comparing original and interpolated or extrapolated motions. In the latter half of this research, the new algorithm is also experimentally examined.
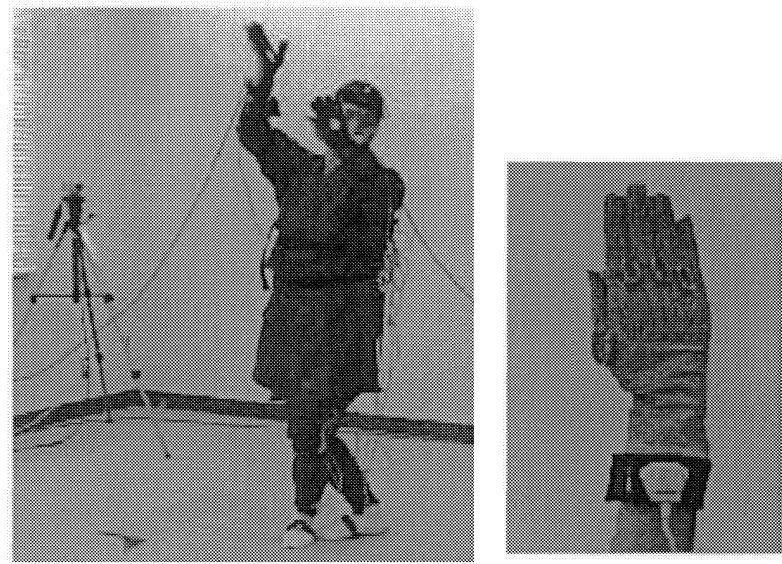

Figure 3. A magnetic motion capture and a data glove

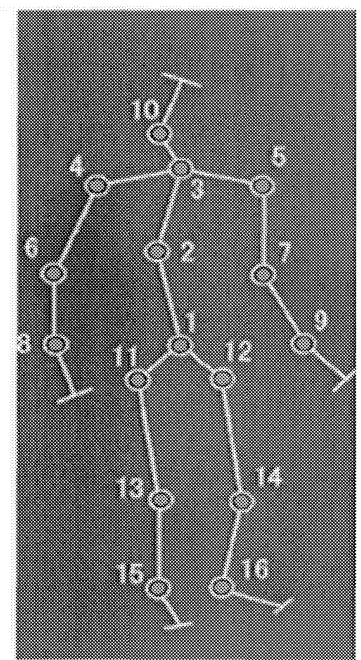

Figure 4. A skeleton model 


\section{Methods}

In this study, MotionStar Wireless (Ascention Technology Corporation) as a magnetic motion capture system and a pair of Cybergloves (Virtual Technologies) as data gloves were used for measurement of human movement shown in Figure 3. The motion capture has sixteen sensors which respectively output six degrees of freedom on the position and direction (i.e. $\mathrm{x}, \mathrm{y}, \mathrm{z}$ coordinates, heading, pitch and bank) by the matrix form. The data glove has eighteen sensors with the same form of outputs as the motion capture. The motion sensors can freely be fixed at the position of the bones of both sides of a joint, by the estimation method of a joint center point described in the following.

In the motion capture system, white noise as the mean of the signal is almost zero and the variation changes in proportion to the distance from a magnet transmitter is overlapped as a system noise [4]. Prior to the main trial, therefore, about twenty-minute outputs are acquired by sufficiently moving subjects' bodies and sensors fixed. The intersection points of each two vectors from the sensors are obtained by least squares method with the pretrial data. Because each radius between the presumed center of the human bones and the surface position with attached sensors is easily calculated with the length of velcro tapes for fixing sensors, the estimation of each joint center is carried out by the abdominal shift of the intersection point by the bone radius. In the meantime, the human skeleton model is beforehand constructed with fifteen joints in the computer, as shown in Figure 4. The information of the joint positions in test subjects is fitted the human model. The length of the bone which is equivalent to the distance between two joints is automatically calculated, does not require the physical measurement. Finally, time-series data in each joint angle is calculated with each joint center point and two sensor outputs neighboring. The degrees of freedom in the joint angles are restricted: three in hip, backbone, neck, shoulder and knee; two in ankle, elbow and wrist.

The algorithm of the dance computer-graphic synthesis is as follows. First, the range of approximately periodic motion for interpolation or extrapolation is decided, where a motion used is approximately a three or four times repeated cyclic movement. Secondly, amplitude spectrum and phase spectrum are obtained from time- series data of each joint by Fourier transformation. Thirdly, one of two patterns of motions is selected as a standard dance pattern, and the other as a comparison. The differences of frequency components in the two patterns are calculated in the frequency domain, where the spectrum extension or compression were adopted so that interpolation or extrapolation of movement can be carried out in two kinds of motions with different fundamental frequencies. Fourthly, according to 
the indication of users, the weight is given to the difference, the weighted component is added to the standard motion in the frequency domain, and a new motion is synthesized. Here, the information of fundamental frequency is also interpolated or extrapolated in the frequency analysis and the spectrum extension or compression is conducted in one of motions for the motion synthesis, so as to interpolate or extrapolate the speed of dance movement. Finally, joint angle time-series data are synthesized by inverse Fourier transformation, with careful operation of the phases in every joint. The computer-graphic animation is synthesized with the time-series data given to the human skeleton model.

\section{Experiments}

The previous researches reported that maximum orders of human impression structure for evaluation of dance are nine [5]: beauty, brightness, vigor, firmness, harmony, weight, complexity, size, and originality, respectively. The most suitable adjective and adverb pairs were therefore chosen for instruction of dance performance from the precedence research [1] as a reference. Nine pairs of the selected adjectives, adverbs, and their negatives were separately and auditorily presented to three Okinawan dance specialists, instructing to perform dance motions which may produce subjective impressions to observers corresponding to each word. In this experiment, the specialists were asked to perform Okinawan folk dance "Kachaasii", where the performer' movement is mainly produced with arms and hands, without any rules of motions or ways of operation, but is moderately periodic which is suitable for engineering analyses.

The examples of computer-graphic synthesis are as follows: Figure 5(a) is "a standard dance" or a dance motion which were performed to give no specific impressions to observers. Figure $5(\mathrm{~b})$ is "a gloomy dance" as a comparison. Then, examples of interpolation and extrapolation of dance motions are shown in Figures 5(c), (d) and (e) with different weights of the feature component, where Figure 5(d) is an example of the interpolation of two motions which may exhibit a little gloominess, and Figures 5(c) and 5(e) are examples of the extrapolation which exaggerate non-gloominess or gloominess as one and a half times as the original motion.

This system is a computer-graphic animation tool which synthesizes one motion with two kinds of motion data. However, our system can theoretically synthesize with more than three motions, because the synthesis is carried out only by adding weighted differences to one motion in the frequency domain. It may also be possible to synthesize a motion which gives the mixed impression of fifty percent of elegance, thirty percent of 
dynamics, and twenty percent of weakness. The synthesis with more than three motions can practically be carried out by repeated processing.
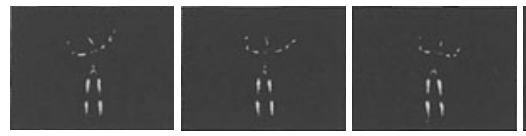

(a) standard dance, $w=0.0$

(b) gloomy dance, $w=1.0$
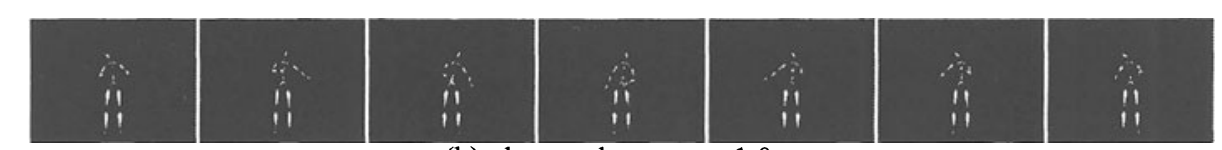
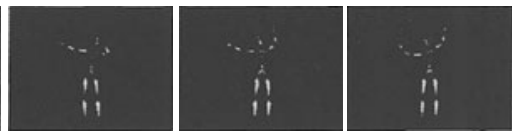

1
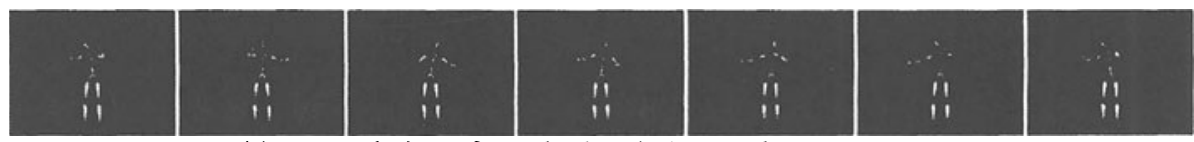

(c) extrapolation of standard and gloomy dance, $w=-0.5$
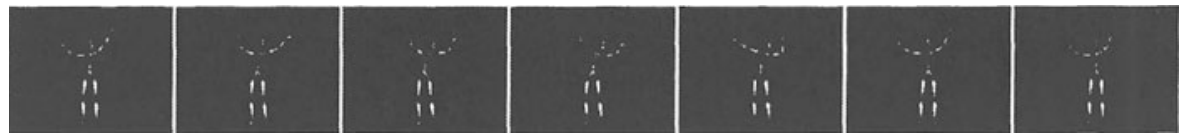

(d) interpolation of standard and gloomy dance, $w=0.5$
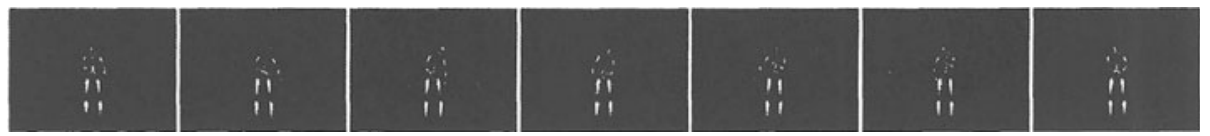

(e) extrapolation of standard and gloomy dance $\mathrm{w}=1.5$

Figure 5. Examples of computer-graphics synthesis

\section{Estimations}

The estimation method of joint center point in this study uses the property in which the temporal average of the noise from a magnetic motion capture system is almost zero, as mentioned above. To verify the validity of the method, the length of the arm was estimated instead of the position of a joint center, because a physical joint center can not be measured in the human body. A multilink with two joints was used for the experiment and both of the joints move to all directions in three-dimension. The multilink, 
however, has the structure in which the joint center point fluctuates according to the motion, as seen in the human muscular-skeleton structure. The ranges from each magnetic sensors to the center of two joints are respectively $21.1 \mathrm{~cm}$. As the results, the estimated ranges with our method were $21.00+1.65$ and $21.09+1.81 ; 21.02+0.78$ and $21.60+1.06$ as a mean (cm) plus S.D. respectively; under two conditions as follows: the multilink was swinging quickly and slowly. The results suggested that quick moving of the multilink a little bit decreases the estimation accuracy. However, it is possible to conclude that our method can obtain a good estimation score even if there may be some fluctuations of sensor position or the system noise in a practical experiment.

\section{Conclusions}

The purpose of this study is to design a computer-graphic animation tool through which the relationship between human motions and subjective impressions can be quantitatively examined. For example, kinematic or dynamic features which may produce the impression of "cheerfulness" or "gloominess" may be possibly analyzed by comparing cheerful and gloomy motions with a $50 \%$ interpolated cheerful motion or $200 \%$ extrapolated cheerful motion.

In this system, the three-dimensional human motion was measured with a wireless magnetic motion capture system with 16 sensors and a pair of data gloves with 18 sensors each. Then, the time-series changes of each joint angle were estimated through the measured data, using a method where each center of the joint angles were estimated based on the stochastic behaviors of the sensors, and where each joint angle was calculated with position vectors from neighboring two sensors and a joint center-point estimated. Then, the behaviors of joint angles were analyzed in the frequency domain. Finally, joint motions were interpolated or extrapolated in the frequency domain between two patterns of dance motions selected at random, and a new motion was synthesized with the inverse Fourier transform and displayed as a computer-graphic animation in the time domain.

As the results, our computer-graphic animation tool can interpolate and extrapolate various pairs of approximately periodic dance motions with high accuracy. The experimental investigation of our algorithm for joint-center estimation also suggested that the time-series changes of the angles can be estimated with high accuracy although the magnetic motion-caputure data include considerable amount of noise. 


\section{Acknowledgements}

This research was supported in part by the Ministry of Education, Culture, Sports, Science and Technology (MEXT), Grant-in-Aid for Scientific Research on Priority Areas (A), Research for Comprehensive Promotion of Study of Brain, and by the research project on "Giga-bit Network Utilization" from Telecommuni-cations Advancement Organization of Japan (TAO).

\section{References}

[1] M.Shiba: "Extraction of Kansei Information in Dance Movements", Proc. ATR Workshop on Visual Communication Environments Bridges over Art/Kansei and VR Technologies, pp.68-88, 1998.

[2] K.Hoshino, S.Kamisato, T.Arakaki: "What Produces "Superimpressions" in Dance: Quantitative Investigation of Relationship between Movements and Impressions", Proc. Workshop on Interactive System and Software 2000, Vol.8, pp.45-50, 2000.

[3] N. Unuma, R. Takeuchi: "A Stray”, NICOGRAPH, Computer Graphics Film Show, 1991.

[4] J.F.O'Brien, B.E.Bodenheimer, G.J.Brostow,, J.K.Hodgins: "Automatic Joint Parameter Estimation from Magnetic Motion Capture Data", Proceedings of Graphics Interface 2000, pp.53-60, 2000.

[5] K.Hoshino, T.Arakaki, S.Odo, S.Kamisato: "CGA system Interpolationg and Extrapolating Human Motions", Proc. Workshop on Interactive System and Software 2001, Vol.9, pp.95-100, 2001. 